\title{
Trans-arterial embolisation therapy of dural carotid-cavernous fistulae using low concentration n-butyl-cyanoacrylate
}

\author{
Ming-Hua Li • Hua-Qiao Tan • Chun Fang • \\ Yue-Qi Zhu • Wu Wang • Jue Wang • Ying-Sheng Cheng
}

Published online: 22 July 2009

(C) Springer-Verlag 2009

Erratum to: Acta Neurochir

DOI 10.1007/s00701-008-0133-0

Section "Materials and Methods", right column, line 15

The line should read

Subsequently, the prepared NBCA/Ethiodol mixture (NBCA, Histoacryl; Braun, Melsungen, Germany)

The online version of the original article can be found at http://dx.doi. org/10.1007/s00701-008-0133-0.

M.-H. Li • H.-Q. Tan $(\bowtie) \cdot$ C. Fang $(\bowtie) \cdot$ Y.-Q. Zhu $\cdot$ W. Wang $•$

J. Wang $\cdot$ Y.-S. Cheng

Department of Radiology, Shanghai Sixth People's Hospital,

Affiliated to Shanghai Jiao Tong University,

600 , Yi Shan Road,

Shanghai 200233, China

e-mail: hzthqiao@163.com 